\title{
EPS and Physics Teachers
}

In the light of restructuring EPS, national physical societies are presently being asked by the Executive Committee if they would be interested in registering some teachers as EPS members, in which case appropriate EPS activities would be developed. Maurice Jacob, the Society's President, discussed opportunities with James Stith, the President of the American Association of Physics Teachers, (AAPT) during the joint American Physical Society (APS)-AAPT 1992 Spring Meeting in Washington, D.C.

The AAPT has some 10000 members of which 4000 teach in colleges. Relatively few high school science teachers are members because only teachers with a first degree in physics tend to join. The AAPT holds three general meetings each year comprising the joint APS-AAPT meeting and two four-day meetings which take place on university campuses. They all involve lectures, special sessions (e.g., computers in education), workshops, and discussions about text books. The AAPT publishes a magazine for physics teachers each month during the school year (nine issues p.a.) as well as the monthly American Journal of Physics on college-level education. The AAPT is associated with the American Institute of Physics so members also receive Physics Today each month.

Dr. Stith thinks a sizable number (2000) of AAPT members belong to APS because APS helps the AAPT act as a focus for information about physics, provides support to high school programmes, analyses high school and college curricula, and promotes affirmative actions in support of minority groups.

With the American experience as a background, the Presidents considered a possible rôle for EPS. One opportunity is to coordinate national society efforts to help with pre-university education, and to complement them with special EPS activities. Extending European Community mobility programmes to schools may call for an enlargement of the scope of the student mobility scheme (see page 100) and of the planned evaluation of physics curricula.

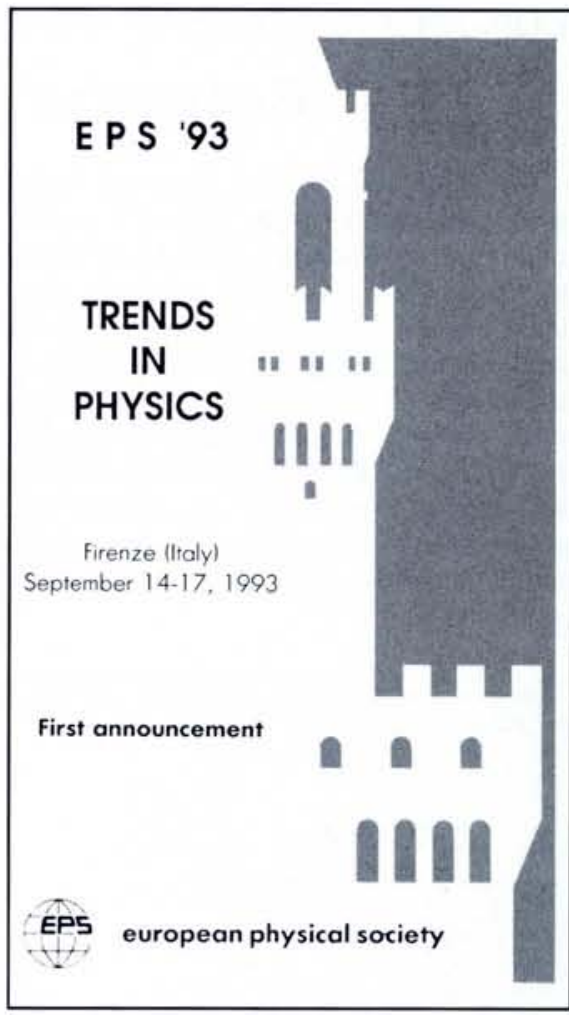

The Eindhoven University of Technology, The Netherlands, tw is a high-tech university.

- The university concentrates on fundamental technological research and high-quality education for future engineers, designers and researchers.

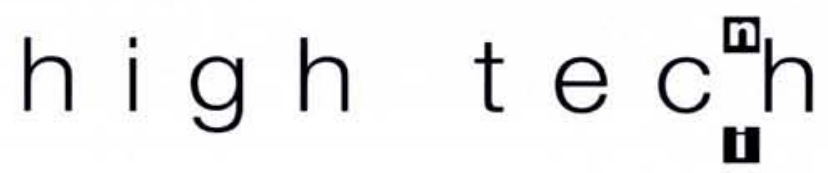

\section{Lecturer in (computational) fluid dynamics}

A regular tenure-track position in Fluid Dynamics at the level of University Lecturer is available at the Fluid Dynamics Group of the Department of Technical Physics of the Eindhoven University of Technology.

The Fluid Dynamics group consists of eight academic staff members and approximately fifteen Ph.D. students, and its research activities concern vortex dynamics, turbulence, aero-acoustics, gas dynamics and heterogeneous media. Within the area of vortex dynamics, the research is mainly aimed at two-dimensional vortices as occurring in rotating and stratified fluids. The present approach is mainly theoretical and experimental (advanced laboratory facilities are available) and it is planned to start up an additional CFD line for the numerical modelling of vortices.

\section{requirements}

We search for an outstanding fluid dynamicist with a strong record of accomplishments and leadership in computational fluid mechanics The candidate is expected to contribute to the vortex dynamics programme, and to develop new research lines within this field. In addition, he/she is expected to have affinity with experimental and analytica research on fluid dynamics in general.

The candidate should contribute to the Departments' general physics and fluid dynamics teaching programme.

\section{information}

Further information on this position can be obtained from the Chairman of the

Fluid Dynamics Group,

Prof. G.J.F. van Heijst, phone $++31-40-472722$ salary

Maximum gross salary is $\mathrm{Hfl} .7528$, per month, depending on the candidate's qualifications and experience.

\section{application}

Letters of application, including curriculum vitae, list of publications and the names and addresses of three references, are to be sent within four weeks after publication of this announcement to: Mr. G.H. Pasmans, Managing Director of the Department of Technical Physics, Eindhoven University of Technology, P.O. Box $513,5600 \mathrm{MB}$ Eindhoven, The Netherlands, with the indication V 34094

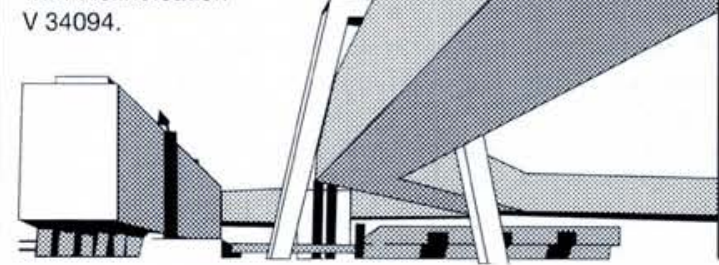

